\title{
Multiple Asymptomatic Spontaneous Coronary Artery Dissections: A Case Series
}

\author{
Mohd Lateef Wani, Cornelia S Carr* and Abdulaziz M Alkhulaifi \\ Department of Cardiothoracic Surgery, Heart Hospital, Qatar \\ *Corresponding author: Cornelia Carr, Cardiothoracic Surgery, Heart Hospital, Doha, Qatar
}

\begin{tabular}{|c|c|}
\hline ARTICLE INFO & ABSTRACT \\
\hline Received: 蔧 April 04, 2019 & Coronary artery dissection is a rare cause of acute myocardial infarction. The clinical \\
\hline Published: 幽 April 12, 2019 & $\begin{array}{l}\text { symptoms can range from chest pain to sudden cardiac death. Most of the patients } \\
\text { with spontaneous coronary artery dissections present with chest pain. Asymptomatic }\end{array}$ \\
\hline $\begin{array}{l}\text { Citation: M Lateef Wani, Cornelia S } \\
\text { C, A M Alkhulaifi. Multiple Asympto- }\end{array}$ & $\begin{array}{l}\text { spontaneous coronary dissection is rare. We present a case series of three patients with } \\
\text { multiple spontaneous coronary artery dissections many of which may have occurred with } \\
\text { no symptoms. }\end{array}$ \\
\hline
\end{tabular}
matic Spontaneous Coronary Artery Dissections: A Case Series. Biomed J Sci \& Tech Res 17(1)-2019. BJSTR. MS.ID.002935.

Keywords: Spontaneous coronary artery dissection

Abbreviations: SCAD: Spontaneous Coronary Artery Dissection; LAD: Left Anterior Descending Coronary Artery; RCA: Right Coronary Artery; CAD: Coronary Artery Disease; PCI: Percutaneous Intervention

\section{Introduction}

Spontaneous coronary artery dissection (SCAD) is a rare condition reported in $0.2 \%-1.1 \%$ of patients undergoing coronary angiography $[1,2]$. At least $70 \%$ of SCAD cases are women and 26 - 38\% of cases occur in late pregnancy, peripartum or postpartum [3]. The clinical presentation of SCAD mainly relates to the extent of myocardial ischaemia at the time of the dissection and may require emergency intervention [1,2]. The left anterior descending coronary artery (LAD) is most commonly involved, followed by the right coronary artery (RCA), the left circumflex $(\mathrm{Cx})$ and the left main (LM) [1,2]. Multi-vessel SCAD is rare but has been reported [2-4]. The underlying pathophysiology is unknown but there is often an absence of the conventional coronary artery disease (CAD) risk factors and it is characterized by an intramural hematoma running between the media and adventitia resulting in compromise of the lumen [1]. Diagnosis is made at coronary angiography and can be confirmed by intracoronary imaging (optical coherence tomography) [5]. Although coronary dissection can occur in patients with atherosclerotic coronary disease, there is a distinct population where dissection is truly spontaneous [1-5]. Several potential risk factors have been reported in addition to female sex hormones, these include increased shear stress, connective tissue disorders and cocaine use [6]. We present our case series of three patients with very similar demographic characteristics and appear to have multiple spontaneous dissections, many of which occurred without symptoms.

\section{Patients}

Case 1

A 43 year old male presented with NSTEMI, which was treated conservatively, with an uneventful recovery. He was of normal height and weight and was of South Indian origin. Risk factor for CAD was smoking for 5 years only. There was no history of previous chest pain, unusual childhood illness or any inflammatory disease. Echocardiography showed an ejection fraction of 55\%. Coronary angiography showed dissection in all the three vessels, with the LAD occluded following the dissection (Figure 1). Autoantibody screening, inflammatory screening and CT scan of chest and abdomen was normal. He underwent CABG with uneventful recovery.

\section{Case 2}

A 42 year old male presented with NSTEMI which was managed conservatively with an uneventful recovery. He was of normal height and weight and was of South Indian origin. Risk factors for CAD were diabetes (1 year), hypertension (1 year) and smoking (5 years). Echocardiography showed an ejection fraction of 50\%. Coronary angiography showed dissections in both the RCA and Cx (Figure 2). The LAD had a significant proximal atherosclerotic lesion. CT scan of chest was normal, and no inflammatory changes noted in other vessels. He underwent CABG with uneventful recovery. 


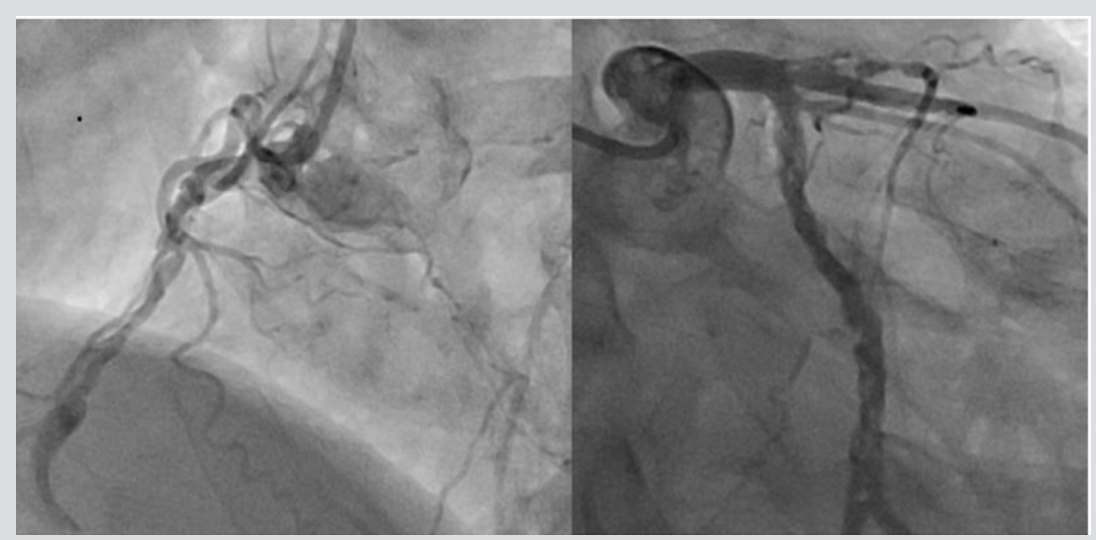

Figure 1: Right injection showing RCA dissection (occluded out of picture) and retrograde filling of LAD (left side); left injection showing Cx dissection and LAD dissection and proximal occlusion (right side).

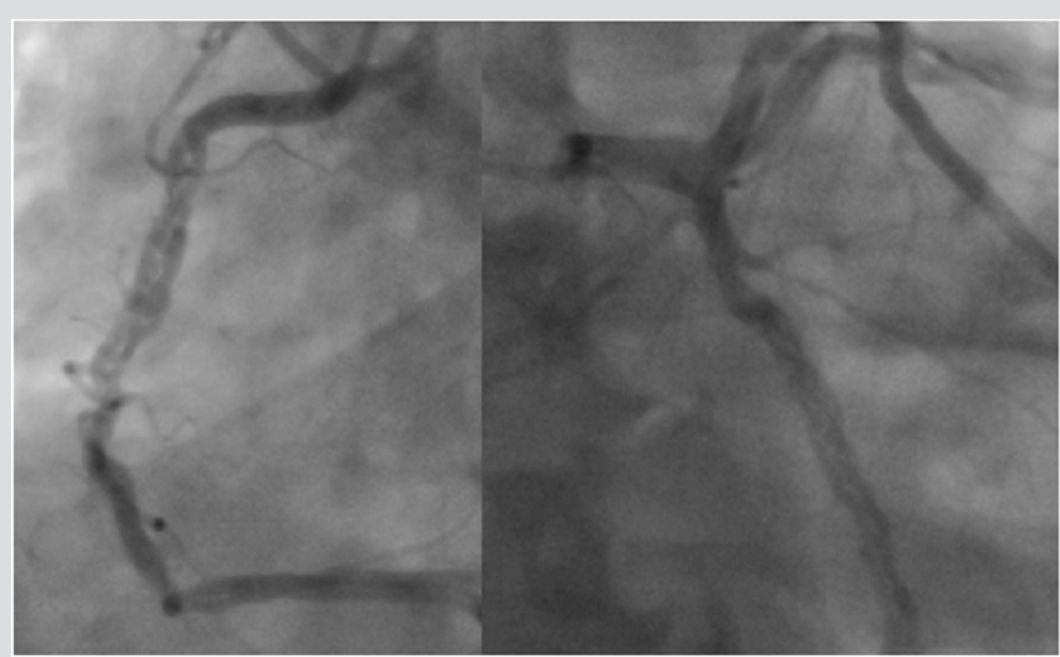

Figure 2: Right injection showing RCA dissection (left side); left injection showing Cx dissection and LAD proximal atherosclerotic lesion at top of image (right side).

\section{Case 3}

A 42 year old male, not known to have any chronic illness with no risk factors for CAD presented with NSTEMI. He was of normal height and weight and was of South Indian origin. His hemoglobin level was $19.4 \mathrm{~g} / \mathrm{dl}$. He was diagnosed as Polycythemia Rubra Vera with a confirmed JAK 2 mutation. He underwent phlebotomy to reduce his hematocrit to below 45\%. Echocardiography showed an ejection fraction of 55\%. He underwent coronary angiography which showed an occluded proximal LAD. The RCA and ramus artery had dissections within them and filled the occluded LAD retrogradely (Figure 3). He underwent CABG with uneventful recovery.

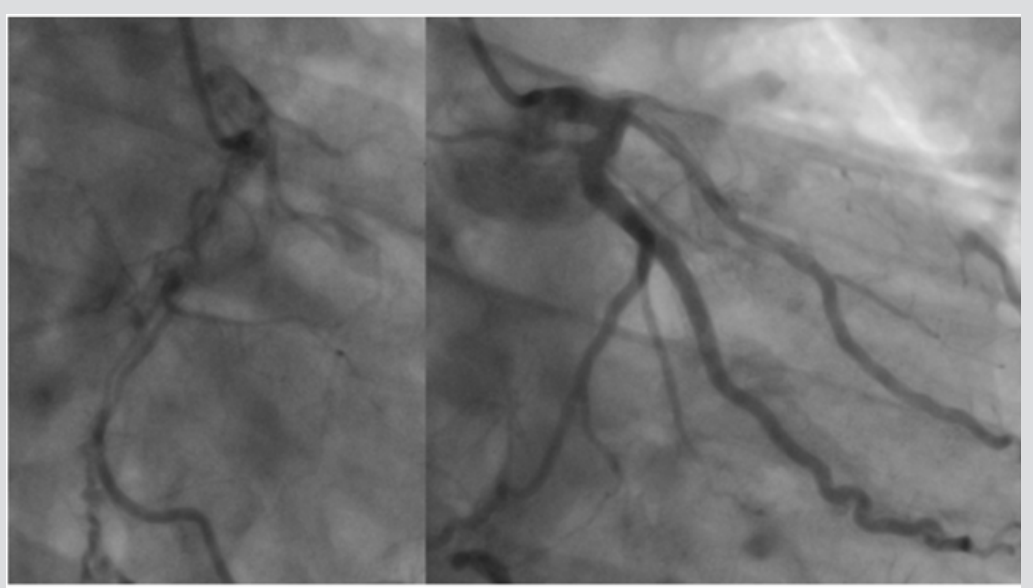

Figure 3: Right injection showing RCA dissection (occluded out of picture) (left side); left injection showing Ramus dissection and LAD occluded with retrograde filling (right side). 


\section{Discussion}

SCAD is well recognized, but rare, and affects a younger, predominantly female population, with a low atherosclerotic risk [1]. There are known predisposing factors $[1,3,5]$ but there are very few cases of triple coronary artery dissection in the literature $[2,4]$, possibly due to the lethality of the condition, but our patients in this case series were completely stable and found to have the lesions on routine coronary angiography. SCAD can be managed conservatively with complete or near complete angiographic resolution in patients who do not have features of ischaemia [4]. Patients who present with compromised coronary flow need revascularization which may require emergency surgery. Percutaneous intervention (PCI) is the most common method to establish coronary flow. Coronary artery bypass grafting is usually reserved for multi-vessel disease, left main involvement or cases where there is on-going ischaemia not amenable to PCI. Our three patients only reported 1 episode of severe chest pain each, yet each had at least two dissections, so some of these had probably occurred without symptoms. All three of our patients underwent CABG because of multivessel occlusions. The patient with polycythaemia may have presented due to thrombosis of a vessel rather than the actual dissection.

All of our three patients had a smooth postoperative period and are doing well on follow up. It is currently unknown if SCAD patients are at risk of other non-coronary events although an increased prevalence of femoral fibromuscular dysplasia has been reported, suggesting the arteriopathy in SCAD may not be localized
[6]. None of our patients have other arteriopathy on evaluation. Spontaneous coronary artery dissection (SCAD) is a rare cause of acute coronary syndrome, but multivessel disease may be more common than previously thought due to some lesions occurring without symptoms possibly as there has been limited reduction in blood flow.

\section{References}

1. Tweet MS, Hayes SN, Pitta SR, Simari RD, Lerman A, et al. (2012) Clinical features, management, and prognosis of spontaneous coronary artery dissection. Circulation 126(5): 579-588.

2. Akgul A, Gursoy M, Bakuy V, Komurcu G, Caglar IM, et al. (2013) Spontaneous triple coronary artery dissection. Ann Thorac Surg 95(4): 1443-1445.

3. Weinberg L, Ong M, Tan CO, Mc Donnell NJ, Lo C, et al. (2013) Spontaneous coronary artery dissection in pregnancy requiring emergency caesarean delivery followed by coronary artery bypass grafting. Anaesth Intensive Care 41(2): 251-255.

4. Backman WD, Bakhai A (2013) Spontaneous triple coronary artery dissection in a 78-year-old woman resolved angiographically with anticoagulation and medical management. BMJ Case Rep pii: bcr 2013008751.

5. Kim Y, Deharo P, Adlam D, Baumbach A, Johnson TW (2017) The role of optical coherence tomography in decision making during the acute phase of spontaneous coronary artery dissection. Int J Cardiol Heart Vasc 14: 6-7.

6. Saw J, Ricci D, Starovoytov A, Fox R, Buller CE (2013) Spontaneous coronary artery dissection: prevalence of predisposing conditions including fibromuscular dysplasia in a tertiary center cohort. JACC Cardiovasc Interv 6(1): 44-52.

\section{ISSN: 2574-1241}

DOI: 10.26717/BJSTR.2019.17.002935

Cornelia S Carr. Biomed J Sci \& Tech Res

(C) This work is licensed under Creative

Submission Link: https://biomedres.us/submit-manuscript.php

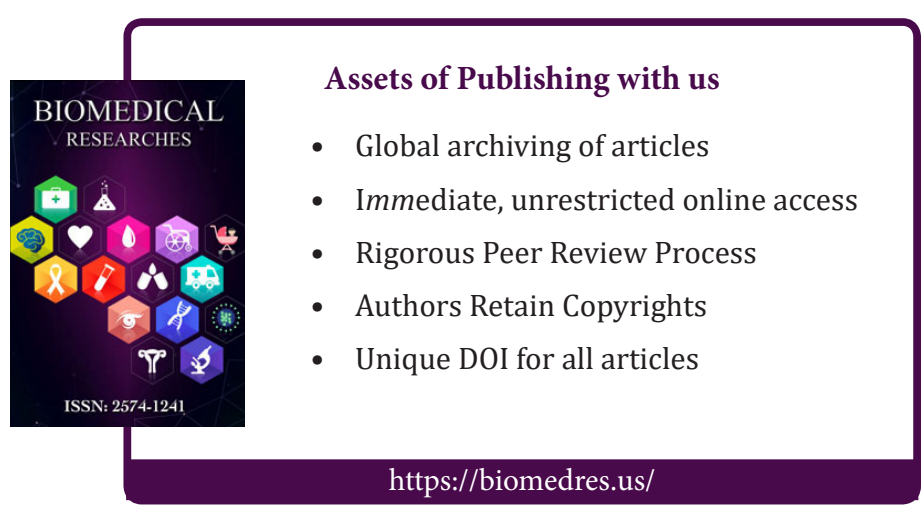

